\title{
Evaluation of an Integral Arising in Numerical Integration Near a Logarithmic Singularity
}

This paper is concerned with the evaluation of

$$
I=\int_{0}^{y} x^{-1} d x \int_{0}^{x} f(u) d u
$$

in terms of values of $f$. If this integral is known, the evaluation of

$$
I_{1}=\int_{0}^{y} f(x) \log x d x
$$

is readily accomplished. For, let

$$
I_{2}=\log y \int_{0}^{y} f(x) d x .
$$

If $f$ is bounded, then

$$
I=-\int_{0}^{y} f(x) \log \frac{x}{y} d x=-I_{1}+I_{2} ;
$$

the two forms for $I$ converge or diverge together. Equation (4) follows from (2) by partial integration. Now the integral in (3) can be approximated by any one of several standard methods. The approximate evaluation of (2) can therefore be achieved, once the evaluation of (1) is completed. In this note coefficients of the Lagrangian type are presented to facilitate the computation of $I$. A rigorous treatment of the error is also presented.

Let

$$
\int_{0}^{r h} x^{-1} d x \int_{0}^{x} f(u) d u=\left(r h / D_{n}\right) \sum_{j=0}^{n} \gamma_{j r}^{(n)} f_{j}+R_{n r}
$$

where the coefficients $\gamma_{j r}^{(n)} / D_{n}$ are such that the remainder $R_{n r}$ vanishes whenever $f(u)$ is a polynomial in $u$ of degree $n$,

$$
D_{n}=\sum_{j=0}^{n} \gamma_{j r}^{(n)}
$$

and $D_{n}$ is independent of $r$, and $f_{j}$ are the tabular values of $f(u)$ at the points $u=j h, j=0(1) n$. Thus, we assume that $f(u)$ may be adequately represented by the $(n+1)$ point Lagrangian polynomial. The interpolating polynomials are easily derived and have recently been published for $n=1(1) 10$ by SALzER [1]. The coefficients $\gamma_{j r}^{(n)}$ and $D_{n}$ are then readily evaluated in a straightforward manner. These are given exactly in Table 1 for $n=1(1) 10, r=1(1)(n+1)$. It is understood that $\gamma_{j r}^{(n)}=0$ if $j>n$, and the $n$ superscript is omitted where no confusion will result. We note some properties of the integrating coefficients useful as a check on the computation.

$$
\begin{aligned}
& \gamma_{j r}^{(n)}=D_{n}, \quad r=j=0 \\
& \gamma_{j r}^{(n)}=0, \quad r=0, \quad j=1,2, \cdots .
\end{aligned}
$$



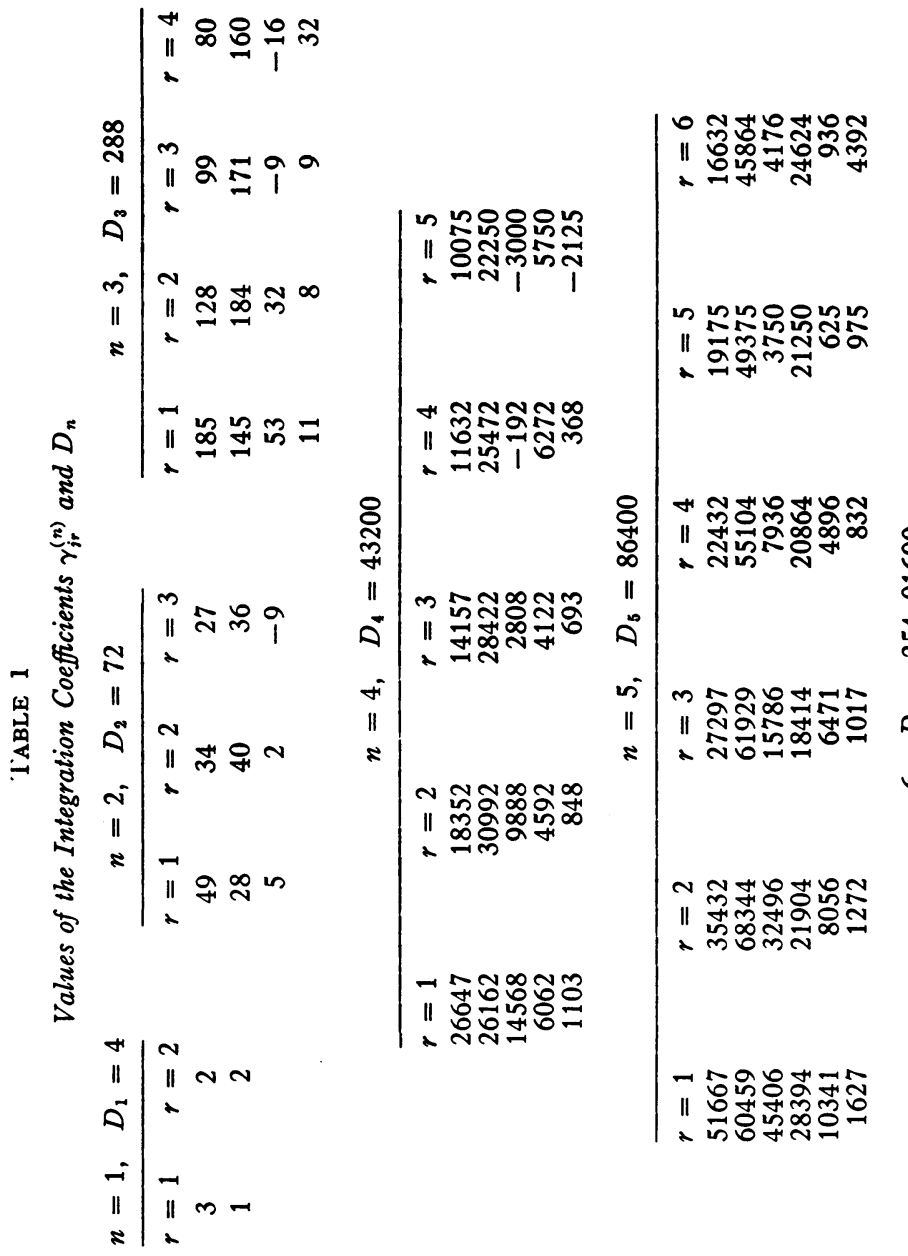

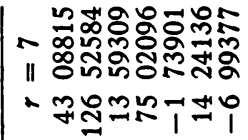

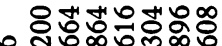

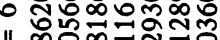

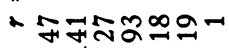

น ำำผำ

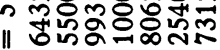

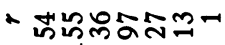

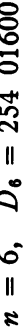

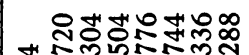

เ

- ชะกำ

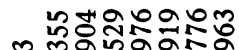

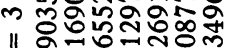

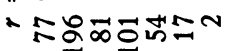

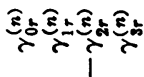

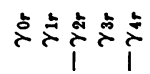

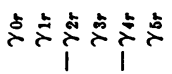

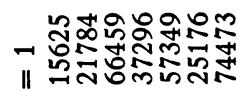

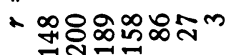




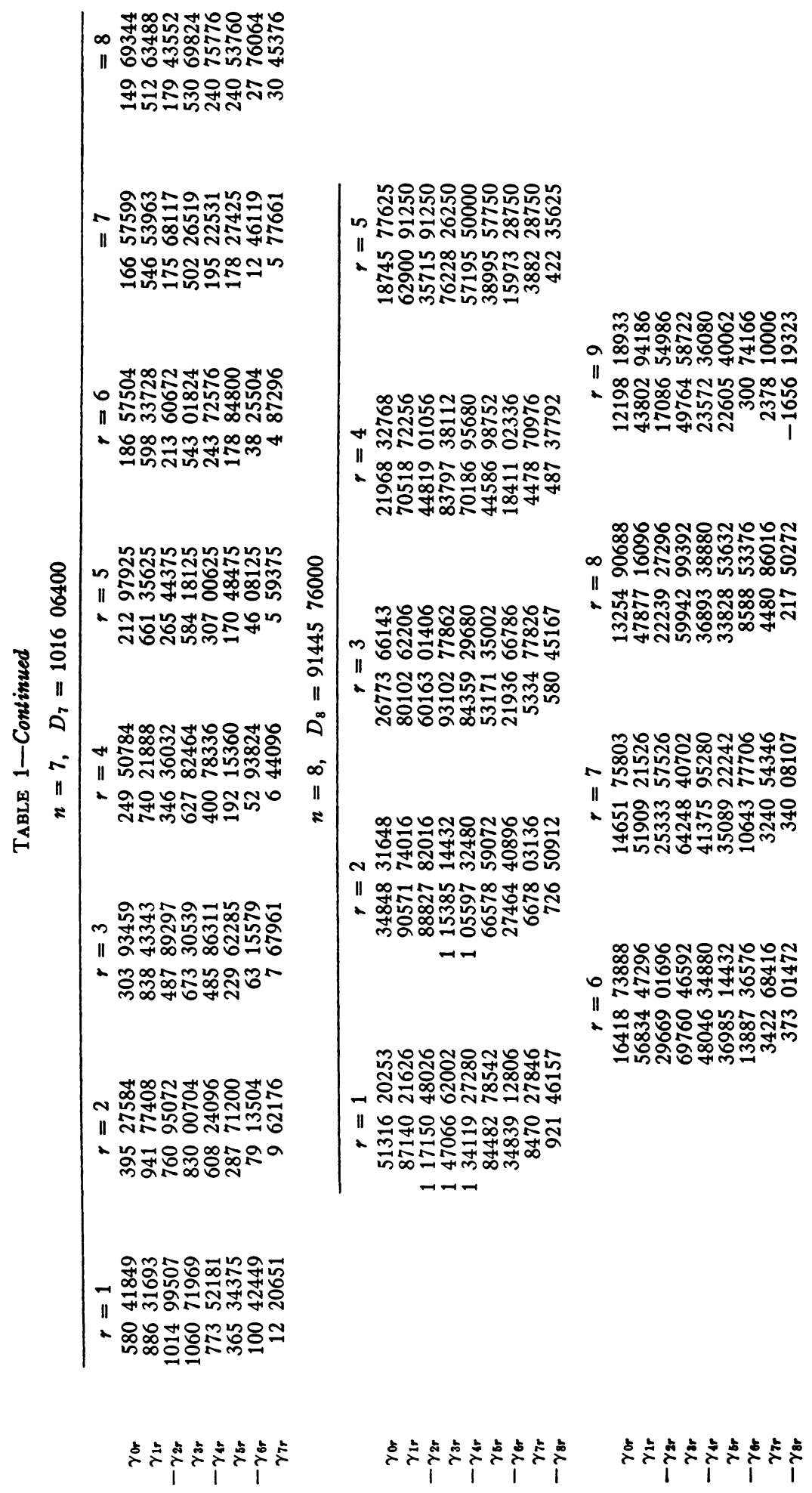




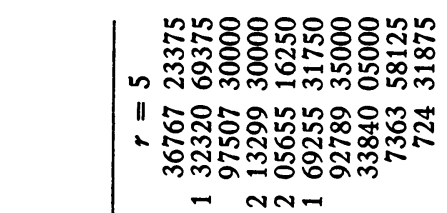

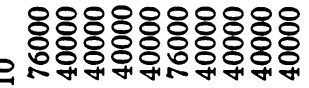

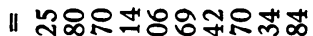

-

- NND

-1

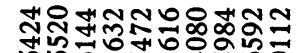
+ ผึ่

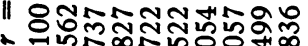

" T-NNT-

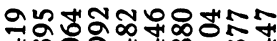

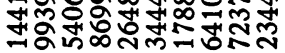

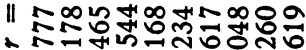

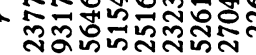

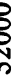

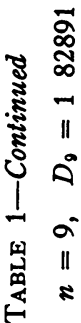

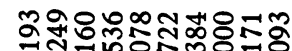

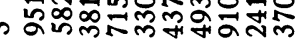

"

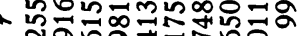

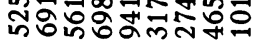

H-NNN-

$r-r$

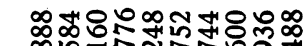

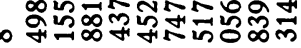

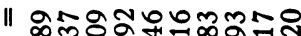

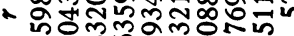

$\rightarrow-r$

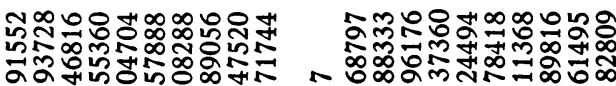
तु్ㅠ유유.

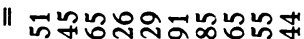

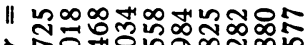

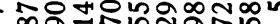

मี่

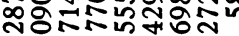

-NMmN

$\rightarrow$ ram

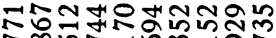

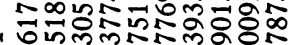

"I 굮ำ

잉 HAntamen

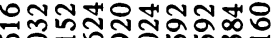

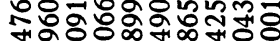

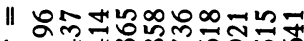

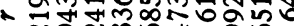

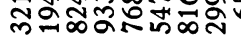

$\rightarrow+$ 


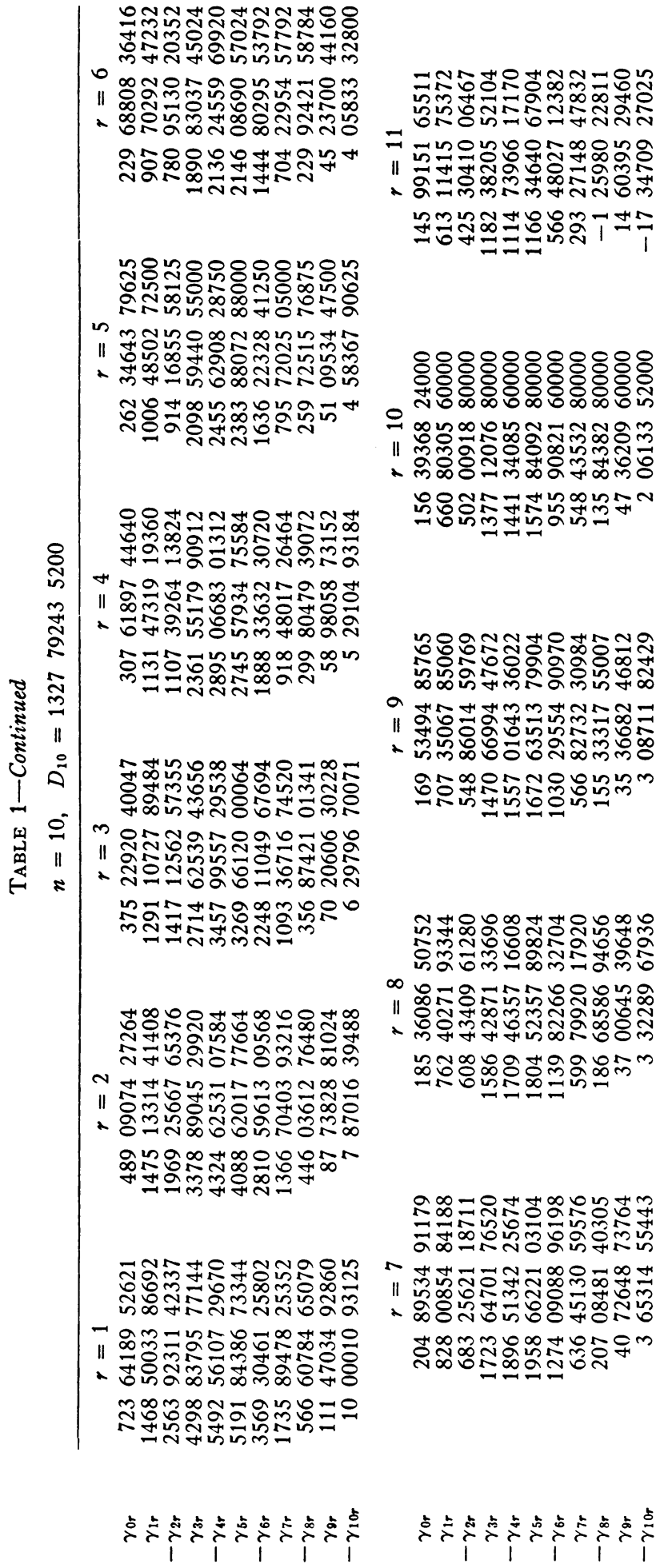


As a function of $r, \gamma_{j r}^{(n)}$ is a polynomial in $r$ of degree $n$ and so the $(n+1)$ th difference must vanish. Hence

$$
\Delta^{(n+1)} \gamma_{j r}^{(n)}(r)=0 .
$$

Thus, employing tables of Lagrangian interpolating coefficients [2], the present entries are easily extrapolated.

Treatment of the error follows the discussion given by MiLne $[3,4]$. Let $c=\max (n h, r h)$. We have

$$
\begin{aligned}
R_{n r} & =\int_{0}^{c} G_{n r}(s) f^{(n+1)}(s) d s, \\
n ! G_{n r}(s) & =\int_{0}^{r h} x^{-1} d x \int_{0}^{x}(\overline{u-s})^{n} d u-\left(r h / D_{n}\right) \sum_{j=0}^{n} \gamma_{j r}^{(n)}(\overline{j h-s})^{n},
\end{aligned}
$$

where

$$
\begin{aligned}
& (\overline{x-s})^{n} \\
& =(x-s)^{n} \text { if } x \geq s \\
& =0 \text { if } x \leq s .
\end{aligned}
$$

Thus $(0, c)$ is the open interval in which $G_{n r}(s)$ does not vanish identically. $G_{n r}(s)$ is said to be definite if it does not change sign in this interval, otherwise it is indefinite. If $G_{n r}(s)$ is definite, the mean value theorem applies and

$$
\begin{gathered}
R_{n r}=E_{n r} f^{(n+1)}(\xi) ; \quad E_{n r}=\int_{0}^{c} G_{n r}(s) d s \\
0<\xi<c . \\
(n+1) ! E_{n r}=r h^{n+2}\left\{r^{n+1} /(n+2)^{2}-\sum_{j=1}^{n} \gamma_{j r}^{(n)} j^{n+1} / D_{n}\right\} .
\end{gathered}
$$

The latter is obtained by putting $f(x)=x^{n+1} /(n+1)$ ! in (5). If we put $f(x)$ equal to the polynomial of degree $n+1$ which vanishes at $x=0, h, \cdots, n h$ and which takes the value $h^{n+1}$ at $x=(n+1) h$ and use equations (5), (11), and (5) with $n$ replaced by $n+1$, we see that

$$
E_{n r}=\left(r h / D_{n+1}\right) h^{n+1} \gamma_{n+1, r}^{(n+1)},
$$

since $R_{n+1, r}=0$. Thus, except for the case $n=10$, the error coefficients are available in Table 1 . The elements for $n=10$ are given in Table 2 . These were obtained by calculating $\gamma_{11, r}^{(11)}$ and checked using (12). They are also interpolable, recognizing that $E_{10, r} / r^{2}$ is a polynomial in $r$ of degree 10 . Since $\lim _{r \rightarrow 0} E_{10, r} / r^{2}=1 / 44$ if $h$ is unity, there are 12 entries, and the requirement that the eleventh difference vanish furnished a further check on the entries. The values were calculated to $7 \mathrm{D}$ and rounded to $5 \mathrm{D}$ for presentation.

The error given by (8) may also be appraised by

$$
\left|R_{n r}\right| \leq \sup \left|f^{(n+1)}(s)\right| \int_{0}^{c}\left|G_{n r}(s)\right| d s .
$$

Alternatively $R_{n r}$ can be expressed in terms of an integral on one of the lower derivatives $f^{(n)}, f^{(n-1)}, \cdots, f^{(1)}$ of $f$. The only essential advantage of definiteness is that it simplifies the evaluation of the integral in (14). 
TABLE 2

$\begin{array}{cc}\text { Values of Error Coefficients } & \text { for } n=10 \\ r & E_{10 r} / h^{12} \\ 1 & 0.00666 \\ 2 & 0.01046 \\ 3 & 0.01257 \\ 4 & 0.01408 \\ 5 & 0.01524 \\ 6 & 0.01620 \\ 7 & 0.01701 \\ 8 & 0.01770 \\ 9 & 0.01834 \\ 10 & 0.01873 \\ 11 & 0.02454\end{array}$

Concerning the definiteness of $G_{n r}(s)$, general theorems are wanting, but in each case definiteness can be checked directly. It has been established that the function is definite for all reported values of $r$ and $n$ such that $r \leq n$. If $r=n+1$, $G_{n, n+1}(s)$ is definite (indefinite) if $n$ is even (odd). In the indefinite cases, $G_{n, n+1}(s)$ has one zero in the open interval $(0, n+1)$. Let $\zeta h$ be the vanishing point. Then the remainder can be decomposed into two parts. Thus,

$$
R_{n, n+1}=f^{(n+1)}\left(\xi_{1}\right) \int_{0}^{\zeta h} G_{n, n+1}(s) d s+f^{(n+1)}\left(\xi_{2}\right) \int_{\zeta h}^{(n+1) h} G_{n, n+1}(s) d s
$$

where $0<\xi_{1}<\zeta h<\xi_{2}<(n+1) h$. The values of $\zeta$ and the two integrals in (15) are recorded in Table 3 for $n=1(2) 9$, mostly to $5 \mathrm{~d}$. The zeros were evaluated by inverse interpolation using TAYLOR's theorem. Availability of the derivatives facilitated the calculation of the integrals.

The heuristic verification that $G_{n r}(s)$ is definite is straightforward, but the numerical work is considerably simplified, especially for $n$ large, using some results due to BARRETT [5]. Let $G_{n r}^{(k)}(s)$ denote the $k$-th derivative of $G_{n r}(s)$ with respect to $s$. He has shown that $G_{n r}^{(k)}(s)$ is defined and continuous in $(0, c)$ closed for $0 \leq k \leq n-1$. With this restriction on $k, G_{n r}^{(k)}(0)=G_{n r}^{(k)}(c)=0$. If $\nu(k)$ denotes the number of zeros of $G_{n r}^{(k)}(s)$ in $(0, c)$ closed, then $\nu(k) \geq k+2$ and $G_{n r}(s)$ is definite if equality holds. If $k=0$, the condition is both necessary and sufficient. For the problem at hand, we first examined the function $G_{n r}^{(n-1)}(s)$ for $n \geq 5$. If $\nu(n-1)=n+1$, then $G_{n r}(s)$ is definite. In a few cases, it was found that $\nu(n-1)=n+2$, and in some of these definiteness was established by showing that $\nu(n-2)=n$. In the remaining cases, $\nu(n-2)=n+1$, and these were

TABLE 3

Values of Zeros of $G_{n, n+1}(s)$ and Error Coefficients for the Indefinite Cases

$\begin{array}{cccc}n & \zeta & \int_{0}^{\zeta h} G_{n, n+1}(s) d s / h^{n+2} & \int_{\zeta h}^{(n+1) h} G_{n, n+1}(s) d s / h^{n+2} \\ 1 & 0.84829 & -0.08602 & 0.03046 \\ 3 & 2.02495 & -0.03961 & 0.00554 \\ 5 & 3.22618 & -0.02661 & 0.00214 \\ 7 & 4.46830 & -0.02001 & 0.00098 \\ 9 & 5.71960 & -0.01602 & 0.00050\end{array}$


shown to be indefinite by examining $G_{n r}(s)$ itself. From the computational point of view, examination of the higher derivatives facilitates the study as the inconvenience of handling large powers of $s$ is removed.

KAPLAN [6] has given coefficients to facilitate the evaluation of

$$
\begin{gathered}
\int_{r_{1} h}^{r_{2} h} g(x) d x \\
g(x)=x^{\eta}[A(x) \log x+B(x)], \quad \eta=0,1
\end{gathered}
$$

in terms of values of $g(x)$. He writes

$$
\int_{r_{1} h}^{r_{2} h} g(x) d x \sim h \sum_{i=\rho}^{\rho+P} c_{i} g(i h)
$$

where $\rho$ and $\rho+P$ are number of tabular intervals $h$ from the singularity to the first and last of group of $P+1$ consecutive ordinates. All formulas are derived on the basis that $A(x)$ and $B(x)$ can be represented by quadratic polynomials. For the most part, the tables can be described as follows. Coefficients $c_{i}$ are given for $\rho=1,5,10$ or 15 and $\left(r_{1}, r_{2}\right)=(\rho+s, \rho+s+1), s=0(1) 4$; also for $\rho=1$ and $\left(r_{1}, r_{2}\right)=(0,1),(0,5)$ and $(0,6)$. Here $P=5$. If $\eta=0$, additional entries are presented for $\rho=1, P=4$ and $\left(r_{1}, r_{2}\right)=(s, s+1), s=0(1) 4$. The latter group are five term formulas and it is assumed that the constant term in the representation for $A(x)$ is missing. Thus, tabular values at the origin are never used. All entries are to $10 \mathrm{D}$. The error term is not considered.

The principal application of Kaplan's table occurs when $A(x)$ and $B(x)$ are not individually known, but $g(x)$ only is tabulated. If each is known, then tables in present paper are more advantageous since representation of $f(x)$ by higher order polynomials is possible. Present tables are useful to integrate out to ten tabular intervals $h$ from the singularity after which ordinary integration formulas may be employed. For integration beyond $10 \mathrm{~h}$, recourse to Kaplan tables may still be appropriate depending on the nature of $f(x)$.

The problem treated in this note is an example of approximate product integration, and has been studied by BEARD [7]. Other examples have been given by SHELDon [8] and Luke [9, 10].

Midwest Research Institute

YUDELL L. LUKE

Kansas City, Missouri

1. H. E. SALZER, "Tables of coefficients for the numerical calculation of Laplace transforms," U. S. Dept. of Commerce, AMS. 30, 1953.

2. "Tables of Lagrangian interpolation coefficients," National Bureau of Standards, Columbia University Press, 1948.

3. W. E. MILNE, "The remainder in linear methods of approximation," NBS, Jn. of Research, v. 43,1949 , p. 501-511.

4. W. E. Milne, Numerical Calculus, Princeton University Press, p. 108-116.

5. W. BARRETT, "On the remainders of numerical formulae, with special reference to differentiation formulae," London Math. Soc., Jn., v. 27, Part 4, 1952, p. 456-464. See also, "On the remainder term in numerical integration formulae," same author and journal, p. 465-470.

6. E. L. KaPlan, "Numerical integration near a singularity," J. Math. Phys., v. 26, 1952, p. $1-28$.

7. R. E. BEARD, "Some notes on approximate product integration," Inst. of Actuaries, Jn., v. 73,1948, p. $356-414$.

8. J. W. Sheldon, "Numerical evaluation of integrals of the form $\int_{\infty}^{\beta} f(x) g(x) d x$," International Business Machines Corporation Seminar, New York, 1950, p. 74-78.

9. Y. L. LUKE, "Mechanical quadrature near a singularity," MTAC, v. 6, 1952, p. 215-219.

10. Y. L. Luke, "On the computation of oscillatory integrals," Cambridge Philos. Soc., Proc., v. 50,1954, p. $269-277$. 